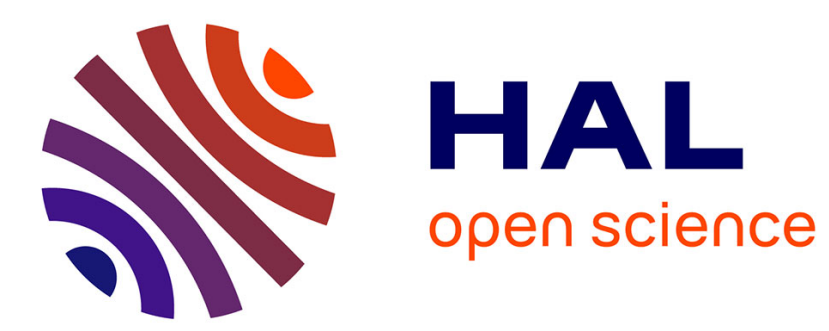

\title{
Biodiversité et politique forestière: défis pour le XXIe siècle
}

\author{
Christian Barthod, Jacques Andrieu
}

\section{To cite this version:}

Christian Barthod, Jacques Andrieu. Biodiversité et politique forestière: défis pour le XXIe siècle. Revue forestière française, 2011, 63 (5), pp.671-677. 10.4267/2042/46113 . hal-03449547

\section{HAL Id: hal-03449547 \\ https://hal.science/hal-03449547}

Submitted on 25 Nov 2021

HAL is a multi-disciplinary open access archive for the deposit and dissemination of scientific research documents, whether they are published or not. The documents may come from teaching and research institutions in France or abroad, or from public or private research centers.
L'archive ouverte pluridisciplinaire HAL, est destinée au dépôt et à la diffusion de documents scientifiques de niveau recherche, publiés ou non, émanant des établissements d'enseignement et de recherche français ou étrangers, des laboratoires publics ou privés. 
CONCLUSION

\title{
BIOdIVERSITÉ ET POLITIQUE FORESTIÈRE : DÉFIS POUR LE XXIe SIÈCLE
}

\author{
Christian Barthod - Jacques Andrieu
}

Chaque article de ce numéro spécial nous a donné à voir une ou plusieurs facettes de ce qui concourt à créer les conditions d'une prise de conscience des enjeux de la biodiversité dans la gestion forestière, puis d'une action respectueuse de la biodiversité, sans dissimuler la pluralité des regards, des analyses et des priorités, voire les contradictions afférentes. Tout en développant quelques axes privilégiés, certains articles se font parfois écho les uns aux autres, mais chacun avec sa coloration spécifique. Toutefois il est également important de prêter attention à ce qui est passé sous silence, soit parce qu'on se trouve dans le domaine de l'évidence ou de l'implicite pour les auteurs, soit parce qu'il s'agit de points particulièrement difficiles sur lesquels les co-auteurs ont eu ou auraient pu avoir des difficultés de partage d'approche, soit encore parce que la complexité de certaines questions aurait nécessité des développements beaucoup trop longs ou complexes pour l'exercice qui leur était demandé. Or certains de ces points passés sous silence, ou évoqués de façon excessivement brève, voire subliminale, tout comme ceux qui traversent plusieurs articles, pourraient bien représenter des défis majeurs pour le XXI ${ }^{e}$ siècle.

L'article de Roland Burrus et Jean-Michel Mourey nous a remis en mémoire deux vérités basiques :

- la préservation de la biodiversité passe par une multitude de décisions individuelles et collectives, certes au niveau des documents de gestion, mais plus encore sur le terrain, et donc par la motivation des propriétaires et gestionnaires forestiers à agir ;

- il existe une dialectique complexe entre une politique de la biodiversité et des décisions individuelles et collectives, entre des pionniers, des réfractaires et ceux qui ne savent pas mais peuvent envisager d'évoluer, entre des discours de structures et la moyenne des comportements.

Dans ce cadre, il est alors possible de s'interroger dans deux directions.

1. La biodiversité ne relevant pas toujours du facilement visible ( $c f$. la biodiversité ordinaire qui est à la base de la fonctionnalité des écosystèmes forestiers), quelles sont les conditions à réunir pour que les décideurs forestiers individuels et collectifs (groupements, associations, coopératives...) aient envie d'agir de manière respectueuse de la biodiversité ? Savoir voir ce qu'il faut voir et en comprendre l'enjeu est une première condition, et il est possible de s'interroger sur la place donnée à l'information et à la formation en matière de biodiversité en forêt par les responsables de la politique de protection de la nature, comme par ceux de la politique forestière (ainsi que par les établissements publics sous leurs tutelles et par les structures qu'ils subventionnent). Mais est-ce suffisant ? Peut-on considérer qu'en prenant conscience du patrimoine naturel présent dans son unité de gestion, le décideur forestier pourra spontanément optimiser ses choix techniques et comparer des options inégales dans leurs conséquences en matière de biodiversité ? Comme le rappellent Catherine et Raphaël Larrère, mais aussi Jean-Philippe Terreaux et Jacques Weber, il n'est pas possible de séparer aisément les grilles d'analyse tech- 
niques et la dimension culturelle et philosophique des choix mettant en jeu la biodiversité. II appartient à la puissance publique de clarifier les messages adressés, explicitement ou non, aux décideurs forestiers. Ce peut être le cas à travers les instruments déployés, incitations budgétaires et fiscales par exemple, qui peuvent promouvoir les actions favorables à la biodiversité ou prévenir les comportements aux effets défavorables. Mais sans doute est-il également nécessaire de développer dans le secteur forestier une ingénierie écologique qui intègre la dimension culturelle sans abuser des raisonnements. Un décideur forestier n'est pas qu'un "Homo oeconomicus », pas plus que seulement un "Homo rationalis ». Dans leur article, Catherine et Raphaël Larrère, en rappelant notamment les préférences esthétiques que les forestiers tentent souvent de dissimuler, invitent à une objectivité qui suppose d'assumer sa subjectivité.

2. La préservation de la biodiversité se raisonne nécessairement à plusieurs échelles spatiales, en fonction des habitats naturels, des espèces et de leur vitesse de déplacement. Bien rares sont les choix relatifs à la biodiversité qui sont pertinents à l'échelle d'une seule unité de gestion forestière, quelle que soit sa taille. La prise en compte des enjeux de connectivité écologique, mais aussi de migration liées aux changements climatiques, confronte de plus en plus souvent les forestiers à l'enjeu du paysage en tant qu'écocomplexe, et donc à la nécessité d'actions qui ne prennent un sens en termes de biodiversité qu'en associant plusieurs (et souvent un grand nombre) de propriétaires fonciers. L'approche reposant sur la seule motivation individuelle touche alors rapidement ses limites. Vouloir gérer la biodiversité au niveau des paysages, comme Sally Collins et Hutch Brown le prônent, mais aussi comme la gestion des sites Natura 2000 (cf. Lucile Rambaud et François Bland) l'exige déjà parfois, nécessite d'innover en termes de processus participatifs et de décision, et souvent de trouver (ou de susciter) des opérateurs compétents à cette échelle, selon une logique que les gestionnaires de la ressource en eau ont expérimenté avant les forestiers. Les élus locaux peuvent jouer un rôle important dans ce domaine, et les maires des communes forestières qui se sont impliqués dans la gestion de sites Natura 2000 à la situation foncière complexe, ou dans des chartes forestières de territoire le savent bien. La gestion de sites d'intérêt patrimonial couvrant plusieurs propriétés (voire plusieurs types de propriétés relevant de statuts juridiques différents) et plusieurs types d'occupation du sol est sans aucun doute un des grands défis forestiers des prochaines décennies. La forêt publique est sans doute mieux armée pour y faire face que la forêt privée. Nos concitoyens sont de plus en plus sensibles à la dimension esthétique du paysage, et les débats sur la biodiversité nous posent de plus en plus souvent la question de la gestion des paysages. Il s'agit d'un débat émergent que les forestiers devront prendre en compte.

L'article de François Lefèvre, Hervé Le Bouler et Bernard Roman-Amat nous a introduit dans un monde climatiquement en évolution, vis-à-vis duquel les gestionnaires forestiers sont nécessairement ambigus. D'une part le débat sur le changement climatique oblige la société à entrer de gré ou de force dans l'univers culturel du long terme que les forestiers revendiquent de fréquenter consubstantiellement, et que les naturalistes appréhendaient peut-être moins. Mais les outils de travail développés au cours des deux derniers siècles par les forestiers concernaient un univers écologiquement stable dans un monde économique et social mouvant. Dans certains pays, le débat a déjà commencé sur la valeur des documents de gestion, pour des raisons qui ne sont pas liées au changement climatique, mais ce dernier risque de ne donner qu'un nouvel argument à ceux qui réclament plus de souplesse dans la gestion pour des raisons écologiques et plus de liberté pour le propriétaire. Les forestiers seraient présomptueux de revendiquer être mieux armés que les naturalistes pour agir, dans leurs domaines respectifs d'intérêt prioritaire. Après avoir longtemps donné le sentiment de considérer que ce qui était bon pour le rendement soutenu en bois ou en grand gibier était nécessairement bon pour toutes les composantes de l'écosystème forestier (théorie de l'effet de sillage), la prise de conscience des enjeux de biodiversité dans la gestion forestière se fait à un moment où les scientifiques spécialistes de la 
biodiversité n'ont souvent pas encore de réponse aux questions découlant des évolutions en cours des écosystèmes et des habitats d'espèces ${ }^{(1)}$. Les forestiers se trouvent donc de plus en plus souvent volontaires pour agir au moment où il est de plus en plus difficile de répondre à leurs questions pratiques. Alors que les espèces sont les éléments les plus visibles de la biodiversité, et que la demande de la société est souvent pressante de ce côté, les gestionnaires forestiers sont confrontés à un dilemme exprimé en 2001 par Botkin : dans un univers aussi mouvant que le nôtre, on peut sans doute conserver la nature en l'état ou conserver les processus naturels en l'état, mais jamais les deux en même temps. Catherine et Raphaël Larrère nous ont rappelé qu'il est possible de voir la biodiversité, soit comme un patrimoine à conserver absolument, soit comme une garantie de l'adaptabilité au changement. Les forestiers auront à choisir la vision dont ils se réclament, à argumenter leurs choix, et à entrer bien malgré eux dans le débat parfois manichéen " espèces versus fonctionnalités écologiques ».

Les réflexions sur changement climatique et biodiversité, développées par François Lefèvre, Hervé Le Bouler et Bernard Roman-Amat, mais aussi les considérations relatives à l'optimisation des choix de gestion, intégrant la biodiversité, qu'ont développées Jean-Philippe Terreaux et Jacques Weber, nous ont sensibilisés au fait que les réponses sont rarement dichotomiques, et qu'il existe plus souvent que les gestionnaires ne le croient (à tout le moins que les " schémas pédagogiques " ne le laissent penser) des gradients et de la place pour un certain pragmatisme. Se pose immédiatement la question de l'échelle de la diversification possible et souhaitable dans une stratégie d'optimisation : la parcelle, l'unité élémentaire de gestion (infrapropriété), la propriété, le paysage, la petite région forestière, la région, etc. ? Sally Collins et Hutch Brown se prononcent clairement en faveur de la maximisation de la biodiversité au niveau des paysages, et pas aux niveaux géographiquement inférieurs. Si l'on accepte le présupposé, autant philosophique que technique, que la gestion forestière, pour prendre en compte de façon raisonnée la biodiversité, peut ne pas chercher la maximisation en tous lieux de la biodiversité, il existe un vaste champ de réflexion pour prendre en charge cette question de l'échelle (spatiale et qualitative) de diversification, intégrant la biodiversité. Mais cela ne peut se décréter a priori par un zonage (2) qui oublierait la diversité des milieux et des propriétaires, mais cela ne peut pas non plus reposer sur le postulat que la somme des décisions individuelles aboutit à l'optimisation à une échelle spatiale supérieure. Si l'on prend au sérieux l'enjeu de biodiversité en politique forestière, cela nécessite de vérifier à différentes échelles la compatibilité entre l'optimisation retenue et les exigences relatives à l'état de conservation favorable des espèces et des habitats, ou des fonctionnalités écologiques, et à créer des outils nouveaux.

De ce point de vue, il convient de prendre en compte l'ambivalence du morcellement foncier forestier, essentiellement en forêt privée, pour la préservation de la biodiversité. D'une part, ce morcellement donne implicitement une certaine garantie minimale, voire moyenne, de conservation d'habitats naturels et d'habitats d'espèces à l'échelle du paysage, même dans les zones à forte potentialité de production économique, car les cahiers des charges des propriétaires sont trop divers pour imaginer la systématisation d'investissements forestiers très intensifs et à très grande échelle, en lieu et place d'écosystèmes forestiers diversifiés ou à forte valeur patrimoniale. D'autre part, mis à part les zones à très faible potentiel de production ou sans desserte forestière possible à un coût économique raisonné par la collectivité, cette grande diversité du cahier des charges des propriétaires fait qu'il ne peut exister en France de vastes zones où la conservation d'habitats naturels ou d'habitats d'espèces serait garantie par la poursuite des sylvicultures traditionnelles. Même si la déprise forestière est une réalité dans certaines zones, et permet de fait la libre expression des mécanismes naturels sur des surfaces non négligeables

(1) Pour des raisons qui tiennent également au fait que les modèles climatiques globaux ne permettent pas de répondre avec un intervalle de confiance raisonnable aux questions relatives à une petite région, et plus encore à une station forestière.

(2) Sauf le cas particulier des espèces ou milieux emblématiques, et reconnues comme telles par un processus respectant certaines formes. 
anciennement exploitées (3), il convient de rappeler que cette situation peut certes présenter des avantages pour la collectivité en termes de " préservation gratuite de certaines composantes de la biodiversité ", mais qu'elle n'est néanmoins pas une panacée, car elle contre-sélectionne certains types de milieux ou certaines espèces, notamment ceux et celles qui dépendent de milieux ouverts en forêt. Une politique de la biodiversité en forêt ne peut jamais faire l'économie de choix et de priorités, et ne peut se reposer seulement sur des positions extrêmes : espérer généraliser en forêt un haut niveau d'interventionnisme au profit de chaque espèce ou habitat à conserver, ou bien s'en remettre à la libre expression des mécanismes naturels pour préserver le patrimoine actuel de biodiversité en forêt. II y a de la place pour des approches à la fois plus structurées et plus nuancées, avec des gradients.

La diversité génétique est le fondement de la biodiversité. Elle en est à la fois la source et une composante essentielle. Il est permis d'espérer que les progrès des connaissances en ce domaine puissent nous apporter un meilleur éclairage sur l'adéquation de nos méthodes de gestion avec nos orientations de politique forestière : les actes de gestion destinés à prendre en compte les évolutions annoncées de l'environnement conduisent-ils réellement les forêts dans le sens voulu ? $\mathrm{Au}$ fond, beaucoup reste cependant à apprendre sur les ressources génétiques des arbres forestiers, mentionnées par François Lefèvre, Hervé Le Bouler et Bernard Roman-Amat.

S’il y a des choix et des priorités à assumer en matière de biodiversité, cela suppose nécessairement une certaine territorialisation des approches. Très rapidement le débat sur la spécialisation des territoires surgit. Il est parfaitement envisageable, et même logique, de voir émerger une " alliance objective » entre ceux qui défendent un choix de ligniculture intensive, et ceux qui prônent un volontarisme ambitieux en matière de politique de la biodiversité : certains peuvent rêver d'un partage des territoires, d'une sorte de Yalta de l'économie et de l'écologie, en oubliant certes la diversité des cahiers des charges des propriétaires et en faisant trop rapidement table rase du patrimoine naturel actuel, très largement réparti et bien présent même dans certaines forêts hautement productives. C'est pour des considérations très pratiques et opérationnelles, liées au "donné forestier et foncier » en Europe, mais aussi au respect de la liberté des propriétaires et gestionnaires forestiers, que le continent européen s'est massivement prononcé en faveur d'une gestion durable multifonctionnelle, dès lors que la multifonctionnalité ne veut pas dire le même niveau d'arbitrage et de priorité entre les différentes fonctions partout. Il est en effet nécessaire et légitime de respecter la diversité des manières dont sont identifiés localement, régionalement et nationalement les enjeux économiques, écologiques, sociaux et culturels liés aux forêts, mais aussi aux biens et services qu'elles produisent. En intégrant les préoccupations relatives à la biodiversité dans la gestion durable, les forestiers européens ont pris un pari qui " fait sens ». Ils se sont notamment engagés à faire évoluer leurs outils traditionnels, notamment leurs documents d'aménagement et de gestion, pour rechercher un certain type d'optimisation des choix de gestion, intégrant la biodiversité, selon la logique exposée par Jean-Philippe Terreaux et Jacques Weber. Ce n'est ni facile, ni rapidement atteignable, mais refuser la logique de spécialisation des territoires implique nécessairement d'accepter de faire un pari de confiance dans les documents de gestion forestière, dans leur forme actuelle, comme dans leur évolution. C'est ce que l'article de Lucile Rambaud et François Bland, comme celui de Henri Plauche Gillon, Jean-Claude Monin et Pascal Viné nous rappellent.

L'article de Guillaume Choumert, Gilles Kleitz et Cyril Loisel, sur la dimension internationale du débat sur la biodiversité dans la gestion forestière, nous introduit dans la manière complexe dont

(3) Cf. la montée des réflexions et débats en Europe sur la " nature férale ", c'est-à-dire le " retour à l'état sauvage » d’une nature autrefois domestiquée. Ce concept relatif aux espèces est en train de s'étendre aux écosystèmes, et notamment aux paysages complexes marqués par la déprise des activités humaines. L'Europe occidentale qui n’a pas conservé de vastes zones forestières peu marquées par l'action de l'homme est particulièrement concernée par ces réflexions, parfois présentées comme une alternative à l'intérêt croissant des populations citadines pour la wilderness. 
le sujet de la biodiversité est en interaction avec le sujet du changement climatique, aux enjeux économiques et politiques perçus comme beaucoup plus immédiats et visibles. Le sujet forestier semble être le lieu où s'affrontent, mais aussi se réconcilient le plus facilement les deux enjeux, avec le mécanisme REDD+ dont la vocation pourrait être de faire une synthèse intégratrice des préoccupations afférentes aux deux conventions internationales, même si cet outil est issu des négociations climatiques et a un objectif "carbone " clairement affiché. Bien que cet outil ne s'applique pas aux forêts européennes, il constitue un précédent a priori intéressant, et les positions françaises en la matière démontrent l'attachement constant des gouvernements successifs à soutenir des outils "poly-usages ", où il est possible de discuter des pondérations données à chaque facteur. La dernière conférence des parties de la convention sur la diversité biologique a mis l'accent sur une ambition renforcée en termes d'espaces protégés au sein de réseaux écologiques cohérents (peu ou prou décliné en France au sein du projet de trames verte et bleue), mais c'est la dynamique communautaire qui traduira sans doute le plus directement au travers de la consolidation du réseau Natura 2000 (cf. Lucile Rambaud et François Bland) et du projet "Green infrastructure " (cf. Robert Flies et Paul Delduc), avec cependant des approches qui ne sont pas réductibles à la forêt. L'incertitude actuelle la plus forte concernant la manière dont les pays du continent européen veulent définir la place dévolue à la biodiversité dans la gestion forestière réside dans l'organisation, les priorités et le calendrier du processus d'Oslo, dans le prolongement de la dynamique des conférences ministérielles sur la protection des forêts en Europe.

Dans les forêts d'outre-mer où réside une part très importante de la biodiversité dont la France est " responsable », Jacques Trouvilliez et Frédéric Mortier nous ont rappelé l'importance des menaces découlant des conséquences de la démographie et du développement économique auquel aspire légitimement la population, mais aussi des espèces exotiques envahissantes, et ceci dans un contexte finalement assez proche de celui des pays en voie de développement. Ceci nous renvoie au passé de nos sociétés européennes, à l'histoire de nos forêts et des pressions dont elles ont été l'objet. La biodiversité est aussi un produit de l'histoire et des équilibres trouvés dans une société. Cette vision sociale et historique de la biodiversité doit nous inciter à prendre conscience des cas où le patrimoine naturel reflète bien un système en équilibre, un héritage dont les conditions actuelles de jouissance sont dans la continuité des conditions qui l'ont fait " prospérer », ou bien un système en transition, au sein duquel se préparent, quasiment à l'insu du gestionnaire, des mutations dont il n'est pas facilement lisible si elles seront ou non positives sous l'angle des préoccupations relatives à la biodiversité. Le débat qui accompagne généralement en France la création d'une aire protégée pour la biodiversité donne souvent lieu à une incompréhension des gestionnaires forestiers : "Si telle espèce ou tel habitat naturel est là, cela devrait être porté à notre actif, et ne pas donner lieu à une "expropriation symbolique" par la puissance publique qui prend la main sur l'avenir du territoire ». Le sentiment d'une forte injustice prédomine, et bien des erreurs ont été faites dans ce domaine, qui ont stérilisé des projets dédiés à la préservation de la biodiversité. L'article de Lucile Rambaud et François Bland montre néanmoins les évolutions en ce domaine, et la manière dont on cherche désormais à prendre en compte cette dimension de patrimoine hérité du labeur des populations locales et d'équilibres ancestraux. C'est bien le moins qui s'impose éthiquement et socialement, mais à la condition de ne pas occulter qu'un tel patrimoine hérité peut être en grand danger, car correspondant à un équilibre révolu, et donc à un état de relique en situation instable. C'est un jugement éthique, philosophique et social qui détermine alors la valeur donnée à ce patrimoine et au prix qu'une société est prête à payer pour s'efforcer de prolonger, par des interventions ciblées, les conditions d'équilibre qui permettent d'espérer un état de conservation favorable de ces composantes de la biodiversité, y compris au prix d'une atteinte au droit de propriété. C'est notamment inévitable lorsque les efforts d'information, de formation et de conviction ainsi que des propositions de contractualisation n'ont pu aboutir, notamment quand il y a incompatibilité entre objectifs et contraintes du propriétaire et de la puissance publique. 
En exacerbant certaines questions et en complexifiant certains débats, le sujet montant des espèces exotiques envahissantes devrait logiquement conduire à devoir clarifier les fondements éthiques, philosophiques et sociaux d'une action en faveur de la biodiversité qui peut coûter très cher. Les stratégies communautaire et française pour la biodiversité font une place grandissante à ce sujet (cf. Robert Flies et Paul Delduc). Il commence à stimuler les réflexions des gestionnaires forestiers dans le monde. Le discours le plus innovant de la séance plénière consacrée à la biodiversité, lors du Congrès forestier mondial de Buenos-Aires (2009), est venu d'un directeur de l'USFS travaillant à Porto Rico, qui a plaidé pour qu'on attache un peu moins d'importance à la question de l'extinction des espèces, et beaucoup plus à celle de l'introduction de nouvelles espèces. Selon lui ce phénomène est d'ores et déjà en train de gouverner l'évolution naturelle de surfaces importantes délaissées par l'homme, selon des modalités beaucoup moins simplistes que ce que le discours habituel laisse entendre : ce sont bien des nouveaux paysages, complexes et auto-organisés qui apparaissent sous l'influence de ces espèces introduites, sans qu'on puisse encore savoir si le nombre et la qualité des services écosystémiques en seront affectés. C'est une question finalement assez voisine du débat forestier récurrent sur la place des essences introduites en forêt, pour laquelle il ne peut être totalement exclu de retrouver des " alliances objectives » du type de celles qui ont caractérisé une partie du XIXe siècle, entre par exemple certains forestiers et ceux des environnementalistes qui privilégieraient nettement les services écosystémiques par rapport au patrimoine spécifique hérité localement de la nature et de l'histoire. C'est une hypothèse que Catherine et Raphaël Larrère n'écartent pas.

On constate l'intérêt fortement croissant pour les services écosystémiques des forêts, au sein d'un vaste débat scientifique et politique dans lequel Bernard Chevassus-au-Louis et Romain Pirard nous ont introduit, après nous avoir sensibilisés aux difficultés sémantiques et méthodologiques. Robert Flies et Paul Delduc nous ont montré la manière dont l'Union européenne et les ministres de l'environnement valorisent une telle approche dans les stratégies communautaires et nationales pour la biodiversité : il s'agit de faire comprendre aux décideurs politiques et aux acteurs économiques, et plus généralement à la société, le " prix de la biodiversité " et le "coût de la non-action ». Mais au travers de cette tentative plus ou moins ambitieuse et plus ou moins achevée de "monétariser la biodiversité ", se joue également une partie de "jeu de go (4) " sur le long terme. Il s'agit notamment de savoir comment assurer efficacement la fourniture de biens publics par des agents privés, comme l'écrivent Jean-Philippe Terreaux et Jacques Weber. L'article de Henri Plauche Gillon, Jean-Claude Monin et Pascal Viné illustre l'enjeu qui s'attache à ce débat, dans un contexte où le modèle économique qui fonde la gestion forestière européenne donne des signes d'essoufflement et peine à se renouveler. Les espoirs fondés sur un possible paiement des services environnementaux (PSE) doivent néanmoins être confrontés au cahier des charges qui semble émerger des expériences qui ont eu lieu dans le monde. Bernard Chevassusau-Louis et Romain Pirard nous rappellent qu'il faut un problème ressenti et une motivation à agir pour que les difficultés à mettre en place un tel schéma soient surmontées : on ne peut pas miser sur une organisation spontanée, les coûts de transaction sont élevés, et le consentement à payer ne dépend pas d'une évaluation économique " objective ". L'implication des autorités publiques est centrale, mais suppose l'identification préalable de ce consentement à payer de certaines parties. Dans les pays tropicaux, l'accès à une eau de qualité et le stockage de carbone ont été les enjeux privilégiés pour lesquels la solution du PSE a été développée, les essais sur la biodiversité ayant été plus rares et moins aboutis. Les forestiers français sont actuellement confrontés à l'absence de problème ressenti par des parties solvables pour résoudre leur problème. Il n'est d'ailleurs pas exclu que l'encadrement législatif et réglementaire forestier

\footnotetext{
(4) Originaire de Chine et formalisé au Japon, il s'agit du plus ancien jeu de stratégie combinatoire abstrait. II oppose deux adversaires qui placent à tour de rôle des pierres noires et blanches sur un " tablier ", tentant ainsi de contrôler le plan de jeu en y construisant des « territoires » qui se comptent en points.
} 
français, par le caractère positif de ses effets sur la production de services écologiques, n'empêche actuellement de satisfaire le cahier des charges d'un PSE. Néanmoins l'article de Sally Collins et Hutch Brown, dans un contexte américain plus proche du contexte européen que celui des pays tropicaux, donne à penser que le recours à certains outils de marché est envisageable, au profit des gestionnaires forestiers.

L'article de François Lefèvre, Daniel Vallauri et Emmanuelle Neyroumande, et celui de Henri Plauche Gillon, Jean-Claude Monin et Pascal Viné mettent tous les deux l'accent sur la volonté d'assumer l'héritage des années 1990-2000 qui ont vu la reconnaissance par tous des enjeux de la biodiversité dans la gestion forestière et l'apaisement de certaines tensions, sur l'accord concernant la légitimité d'une forêt productive prenant en compte les préoccupations de biodiversité, sur le besoin de dialogue entre forestiers et environnementalistes, et sur l'enjeu de structurer la gouvernance de la biodiversité en forêt. Mais, derrière ces aspirations partagées, se manifeste de part et d'autre le rappel de principes ou de priorités dont il est peu probable qu'ils soient automatiquement consensuels. C'est dire qu'il y a place pour l'explicitation des grilles d'analyses, pour des approches plus territorialisées et pour des compromis, à tous les niveaux géographiques pertinents. Mais la fragilisation du modèle économique forestier, par son incapacité de financer toutes les ambitions à partir des ventes de bois, dans le contexte d'une rigueur budgétaire durable, pourrait crisper certaines discussions sur la biodiversité en forêt. II n'est pas facile de dessiner le nouvel équilibre de la politique forestière, ni la manière dont elle prendrait en compte la biodiversité.

Les tendances lourdes à l'œuvre pour ce qui concerne la biodiversité en forêt depuis au moins vingt ans demeurent : poids croissant des politiques communautaires, directes et indirectes, aspirations environnementales des populations citadines, rôle croissant des collectivités territoriales et des outils du code de l'Urbanisme... S'y rajoutent les entrées complexes liées au changement climatique à la fois sous l'angle de l'adaptation et sous celui de l'atténuation, des perspectives d'évolution lourdes des marchés du bois mondialisés et de nouvelles interrogations quant à l'intégration de cette biodiversité dans la gestion forestière. Plus que jamais, le secteur forestier redoute d'être écartelé entre des tendances centrifuges, bien loin du modèle intégrateur qu'a bâti le Code forestier et qu'il poursuit au travers du concept de gestion durable. Mais il n'est pas exclu que la question des services écosystémiques ne soit le lieu d'un nouvel accord et d'une synergie entre forestiers et société civile, permettant des nouveaux financements, publics ou privés issus d'outils de marché, et d'un nouveau développement forestier fondé en partie sur la reconnaissance de sa valeur écologique.

Christian BARTHOD

Conseil général de l'écologie

et du développement durable

Commission permanente des ressources naturelles MINISTÈRE DE L’ÉCOLOGIE ET DU DÉVELOPPEMENT DURABLE, DES TRANSPORTS ET DU LOGEMENT Tour Pascal B Bureau 20.23

F-92055 LA DÉFENSE Cedex (christian.barthod@developpement-durable.gouv.fr)
Jacques ANDRIEU

Sous-directeur de la forêt et du bois

Direction générale des politiques agricole, agroalimentaire et des territoires MINISTÈRE DE L'AGRICULTURE, DE L'ALIMENTATION, DE LA PÊCHE, DE LA RURALITÉ

ET DE L'AMÉNAGEMENT DU TERRITOIRE 19 avenue du Maine

F-75732 PARIS Cedex 15

(jacques.andrieu@agriculture.gouv.fr) 